\title{
From the Editors' Desk: Peer Review Now and in the Future
}

\author{
Richard L. Kravitz, MD, MSPH ${ }^{7}$ and Mitchell D. Feldman, MD, MPhil \\ 'Division of General Medicine, University of California at Davis, Sacramento, CA, USA; ${ }^{2}$ Division of General Internal Medicine, Department of \\ Medicine, University of California, San Francisco, CA, USA.
}

$\mathrm{J}$ Gen Intern Med 26(12):1385-90

DOI: $10.1007 / \mathrm{s} 11606-011-1901-8$

(c) Society of General Internal Medicine 2011

A t Jon Stewart's rally to restore sanity, held in Washington, DC in October 2010, this sign was prominently displayed (Fig. 1). While we do not recognize the holder of the sign, we would not be surprised if he turned out to be a journal editor. Journal editors disagree on practically every topic imaginable; peer review is the rare exception. To editors, careful prepublication scrutiny of findings by informed peers is an article of faith and a bedrock of scientific progress.

To what degree is faith in peer review warranted? Perhaps not so much as medical journal editors would like. For one thing, peer review rarely intercepts outright fraud. As former New England Journal editor Arnold Relman put it, "Science is at once the most skeptical of activities and also the most trusting.... It is intensely skeptical about the possibility of error, but totally trusting about the possibility of fraud." For another, peer reviewers agree with each other much less than one would hope. An analysis of 5881 reviews of 2664 manuscripts submitted to JGIM (Kravitz et al. PLoS One 2010) suggested that achieving adequate levels of inter-reviewer reliability $(>0.8)$ would require 20 independent reviews, not the $2-3$ that most journals typically solicit.

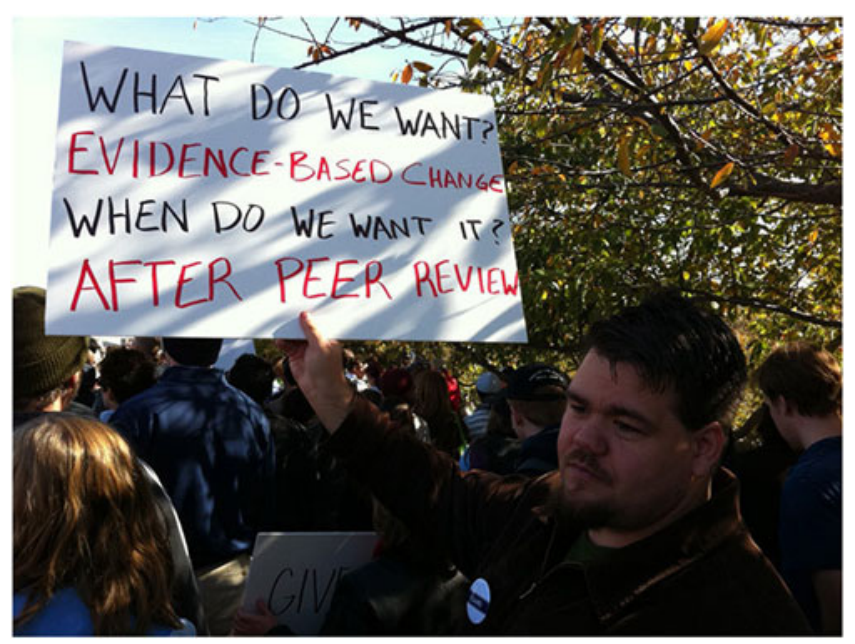

Figure 1. Sign prominently displayed at Jon Stewart's rally to restore sanity, held in Washington, DC in October 2010.

Published online October 5, 2011
On the other hand, a 2007 Cochrane Review concluded that editorial peer review appears to make papers more readable and improves the general quality of reporting. And in an analysis of 507 original research articles submitted to JGIM, articles accepted after peer review garnered substantially more citations than articles rejected and subsequently published elsewhere (Jackson et al., PLoS One 2011). Apparently, even when they disagree, JGIM's peer reviewers contribute measurably to improved editorial decision making by our editorial team (as judged by the admittedly narrow—and potentially biased-metric of subsequent citation rates).

Despite its shortcomings, editorial peer review is here to stay. Our faith in the process depends on the support of an outstanding group of reviewers. In 2010-11, JGIM reviewers volunteered their time and expertise to review about half of the more than 1,000 manuscripts that JGIM receives each year. During this period, 905 reviewers provided a total of 1,143 reviews with a mean quality score of 4.2 on a scale of 1-6 (as judged by JGIM deputy editors). Of these, 213 provided at least two reviews and 23 provided three or more. We are indebted to them for their service.

Among this group of dedicated peer reviewers, there is a cohort that stands out. Reviewers included in this prestigious group performed at least two reviews between July 2010 and June 2011, returned all reviews within 30 days, and received a quality score of four or greater on all reviews. An asterisk identifies the 100 reviewers meeting these criteria. We thank them for their efforts on behalf of the journal.

What about the future of peer review? While pre-publication peer review will remain important, post-publication peer review will gain much more emphasis. As argued by Schriger and Altman (BMJ 2010), "Post-publication critique, as the final arbiter of the meaning of each new communication, is no less important than the earlier phases and is a sign of a healthy scientific community...." At JGIM over the coming months, we hope to advance post-publication peer review in two ways. First, beginning in late 2012, we will begin publishing "mini editorials" alongside selected JGIM articles. These "Capsule Commentaries" will highlight methodological or content issues deserving additional attention, discussion, or debate. Second, we will take advantage of exciting changes in SGIM's web presence to encourage much more interaction among authors and readers. The Society is planning a major web upgrade in Spring 2012. JGIM will have its own virtual space within the SGIM site, offering many opportunities for enhanced interactivity among JGIM's social network. As these changes unfold, we look forward, as always, to your suggestions and feedback. 
Abbo, Elmer

Abou-Raya, Anna

Acheson, Louise

Adegbembo, Albert

Adler-Milstein, Julia

Afable-Munsuz, Aimee

Agrawal, Varun

Agyemang, Charles*

Akrawinthawong, Krittapoom

Akrawinthawong, Krittapoom*

Albright, Karen

Alford, Daniel

Algotar, Amit

Alpert, Joel

Altman, Barbara

Alvanzo, Anika

Ananthakrishnan, Ashwin*

Anderson, Heather

Anderson, Wendy*

Androwich, Ida*

Antommaria, Armand*

Ardemagni, Enrica

Armistead, Niti

Arnold, Louise

Arora, Vineet

Astin, John

Atallah, Sylvana

Attridge, Russell*

Ayres, Lioness*

Baggett, Travis

Bakris, George

Bancroft, Marjory

Bannuru, Raveendhara*

Barkley, Geoff

Barkoudah, Ebrahim

Barnato, Amber

Barr, Michael

Barrett, Brendan

Barrett, James*

Barron, Beth

Barry, Michael

Bassett, Ingrid

Batch, Bryan

Bau, Ignatius

Bauer, Heidi

Baum, Michael

Beach, Mary Catherine

Beck, John*

Becker, William

Befort, Christie

Behler, Caroline

Bell, Ralph

Bell, Robert

Bell, Tanvir

Benard, Vicki

Benin, Andrea

Benner, Joshua

Berg, Jessica

Berg, Katherine
Berger, Barbara

Berkenblit, Gail

Berkman, Nancy

Bernabeo, Elizabeth

Bernstein, Kyle

Berry, Stephen*

Beyth, Rebecca*

Binswanger, Ingrid

Biros, Michelle

Bishop, Tara*

Bix, Laura

Blackall, George F

Block, Jon

Blum, Alex

Bodenheimer, Thomas

Bohannon, Richard

Boltri, John

Bonney, Loida

Bott, David

Boulware, L. Ebony

Boutin-Foster, Carla

Bovet, Pascal

Boyd, Cynthia

Branch, William

Brauner, Daniel

Brendel, Rebecca

Brenner, Darren

Brett, Belle

Briesacher, Becky

Brittain, Kelly

Brody, Howard*

Brophy, Jay

Brotman, Daniel J.

Brunetti, Natale Daniele

Bruns, Anke

Bulchandani, Deepti

Burchell, Ann

Burgio, Louis

Burke, Michael

Burton, Christopher

Bynum, Julie

Cadigan, Jean

Caines, Laurie

Cappelleri, Joseph

Card, Andrea

Carlson, Charlotte

Caron, Aleece

Carrico, Adam

Carrier, Emily

Carroll, Norman

Carroll, Patrick

Carson, Michael

Carter, Michele

Cartwright-Smith, Lara

Cavanaugh, Kerri

Cecchi, Enrico

Cecchini, Michele*

Cegala, Donald

Cene, Crystal
Chakraborty, Subhankar

Chao, Samantha

Cheever, Laura

Chen, Huiju

Chen, Judy

Cheng, Christine

Cheng, Hugo

Cherkin, Dan

Cherrington, Andrea

Cheungpasitporn, Wisit*

Chewning, Betty

Chin, Jeanette*

Chin, Marshall

Choi, Won

Chou, Calvin

Choudhry, Niteesh

Chren, Mary Margaret

Christianson, Jon

Chudgar, Saumil

Chung, Sukyung

Clancy, Dawn

Clark, Alexander

Clark, Cheryl

Clark, Michael

Cochran, Nan

Cody, Marisue

Coffey, Jr., Charles

Cohen, Beth

Cohn, Felicia

Coleman, Kimberly

Comsa, Mihai

Conner, Douglas

Conwell, Leslie*

Cooperberg, Matthew

Cordasco, Kristina

Correa, Ricardo

Correa, Ricardo

Cotton, Sian*

Coups, Elliot

Covinsky, Kenneth*

Crandall, Sonia

Croghan, Thomas*

Cunningham, Peter

Cunningham, William

Cutrona, Sarah*

Cutrona, Sarah

Czaja, Sara

Dacha, Sunil

Daley, Christine

Damiano, Peter

Dandu, Madhavi*

Danis, Marion

Datta, Santanu

Davidoff, Amy

Davis-Smith, Monique

Day, Gregory

Deamant, Catherine*

Dean, Nathan

Degruy, Frank 
Desalvo, Karen

Desroches, Catherine

Dezee, Kent

Dhurandhar, Anjali

Diamond, Lisa

Dibonaventura, Marco

Dieckhaus, Kevin

Diehl, Andrew

Divine, George

Dixon, Brian

Djuricich, Alexander

Dobscha, Steven*

Doering, Tracey*

Doggett, David

Dohan, Daniel

Dolan, James

Dolovich, Lisa

Domino, Frank*

Donaldson, Molla

Dong, Yue

Donohue, Julie

Dotson, Vonetta

Doubeni, Chyke*

Downing, Steven

Du, Xianglin

Duggan, Ashley

Dunton, Genevieve

Duru, O. Kenrik

Dwyer, Johanna

Dyrbye, Liselotte

Edlund, Mark

Effiong, Andem

Eggers, Paul

Eiser, Arnold

Ell, Kathleen

Elliott, Daniel

Elmore, Joann

Elnicki, Michael

Elstein, Arthur

Enderle, Mark

Engelman, Kimberly

Ephrem, Georges

Eslami, Saeid

Fancher, Tonya

Farnham, Paul G.

Farzanfar, Ramesh

Faseru, Babalola*

Fauli, Siri

Federman, Alex

Federman, Douglas

Feero, W. Gregory

Feldman, Roger

Felix, Kaytura

Feng, Qiushi

Feng, Yongqing

Fernander, Anita

Fickel, Jacqueline*

Filardo, Giovanni

Filice, Gregory

Fingerhood, Michael

Finkelstein, Joseph
Fish, Laura

Fisher, Joslyn*

Fishman, Paul

Fletcher, Kathlyn

Floch, Martin

Flores, Ann Marie

Fong, Tamara

Forjuoh, Samuel

Forman, Daniel

Forrest, Christopher

Fortney, John*

Fourtounas, Costas

Fowler, Floyd

Fox, Rena

Fraenkel, Liana

Francis, Maureen

Frank, Erica

Freiberg, Matthew

Friedman, Maria

Friedman, Susan

Fullerton, Stephanie Malia

Fung, Constance

Gabbay, Ezra

Gagnon, Marie-Pierre

Gallagher, Teresa

Gardiner, Paula

Gellad, Ziad

Germain, Michael

Gerrity, Martha

Gertz, Alida

Gibbons, Melinda

Gimm, Gilbert

Ginosar, David

Girotti, Jorge

Glassman, Peter

Gliatto, Peter

Glover, Jackie

Gluud, Lise Lotte

Goddard, Andrew

Goldsmith, Laurie

Gollust, Sarah

Gomez, Arthur

Good, Chester

Goodlin, Sarah

Goodman, Kenneth

Gottlieb, Amy*

Gowd, B.M.Pampana

Grande, David

Grau, Lauretta

Greene, Jeremy

Griggs, Jennifer

Griswold, Kim

Groopman, Jerome

Grothaus, Louis

Guandalini, Stefano

Guddati, Achuta

Guerra, Carmen

Guessous, Idris

Gundareddy, Venkat*

Gusmano, Michael

Günald, Meral
Haaz, Steffany*

Haddad, Mark

Hahn, Elizabeth

Hahn, Steven

Halamka, John

Halfon, Patricia

Hall, Karen

Hall Long, Kirsten

Hallenbeck, James

Hamieh, Tarek

Han, Beth

Hanchate, Amresh

Handler, Steven

Hanoch, Yaniv

Hardy, Susan

Harpole, Linda

Harrity, Shawn*

Hasnain-Wynia, Romana*

Hastings, Susan

Hatahet, Mohamad

Hatala, Rose

Hausmann, Leslie

Hawley, Sarah

Hays, J Taylor

Hazlet, Thomas

He, Jianghua

Hellström, Amanda

Helmer, Drew

Hensley Alford, Sharon

Hepner, Kimberly

Heritage, John

Hermer, Laura

Hernandez, Jose Luis

Heslin, Kevin

Hess, Rachel

Hesse, Bradford

Hester, D. Micah

Hewett, David

Hicks, Frank

Hicks, Leroi

Higgins, Stephen

Hinch, Bryan

Hinchey, Kevin

Hinote, Brian*

Hirsh, Joel

Hirshfield, Sabina

Ho, Michael

Hodgkin, Dominic

Hoellein, Andrew

Hoenig, Helen

Hoffman, Richard

Hogan, Timothy

Holden, Debra

Holl, Jane

Holmes, Ann M.

Holt, Cheryl

Holt, Stephen

Holt, Stephen

Horvitz-Lennon, Marcela

Hsiao, Chun-Ju

Huggett, Kathryn 
Hui, Sk Azor*

Humphreys, Keith

Hunter, Christopher

Hwang, Stephen

Iftikhar, Salma

Imazio, Massimo

Insinga, Ralph

Irani, Farzan

Jaar, Bernard

Jackson, Christian

Jackson, George

Jacobson, Janet

Jacobson, Peter

Jaeger, Jeffrey

Jamal, Yasser

Jean-Jacques, Muriel

Jelley, Martina

Jennings, Ian

Jenq, Grace

Jhamb, Manisha

Johl, Karnjit

Johnson, William

Johnston, Doug

Jonas, Wayne

Jones, Mark

Jones, Resa

Jordan, Lanetta

Joseph, Djenaba

Jureidini, Jon

Juul, Dorthea*

Kado, Deborah

Kaestner, Robert

Kahan, Scott

Kahlon, Gunjan

Kahn, Katherine*

Kaiser, Karen*

Kaissi, Amer

Kalager, Mette

Kanade, Sandhya

Kane, Robert

Kane-Gill, Sandra

Kaphingst, Kimberly*

Kaplan, Sherrie

Kapo, Jennifer

Kapoor, Alok

Karan, Mehmet Akif

Karliner, Leah

Karter, Andrew

Kassab, Maria

Kassirer, Jerome

Kattan, Michael

Kaufman, David

Kavalieratos, Dio

Kavanaugh, Megan

Keenan, Patricia

Kehle, Shannon

Kertesz, Stefan*

Kesselheim, Aaron

Kessler, Chad

Ketcham, Jonathan

Keyhani, Salomeh

Kilbourne, Amy
Kilgore, Meredith

Kilo, Charles

Kim, Theresa

Kimberg, Leigh

Kimerling, Rachel

King, Diane

Kinney, Eleanor*

Kizer, John

Kleshinski, James

Klugman, Craig

Knight, Chris

Knight, Kelly

Knight, Sara

Kogan, Jennifer

Koracevic, Goran

Korenstein, Deborah

Korthuis, P. Todd

Kosteniuk, Julie

Kotla, Sumankrishna

Kovner, Christine

Kramer, Jennifer

Krebs, Erin*

Kremer, Heidemarie

Krishnan, Ranjani

Kroenke, Kurt

Kronish, Ian

Kullgren, Jeff*

Kullgren, Jeffrey

Kumagai, Arno

Kumar, Ambuj

Kumar, Nilay

Labarère, José

Lagu, Tara

Lamantia, Michael

Landman, Adam

Landon, Bruce

Lantos, John

Latham, Stephen

Lavela, Sherri*

Lazarus, Cathy

Legare, France*

Leigh, J. Paul

Leland, Natalie*

Lemieux, Josh

Lenhard, M. James*

Lerfald, Nathan

Levin, Theodore

Levinson, Wendy

Lewis, Lisa

Lewis-O'connor, Annie

Leykum, Luci

Li, Jun

Li, Yuyuan

Liberman, Joshua*

Lieberman, Steven

Liebow, Mark

Like, Robert

Lin, Grace

Lin, Ming Valerie

Linas, Benjamin

Linden, Judy

Lindenauer, Peter
Lindenberger, Elizabeth

Lio, Peter

Lo, Margaret

Lo Sasso, Anthony

Loeb, Danielle

Lotufo, Paulo

Lowry, Becky*

Lu, Lawrence

Lunze, Karsten

Ma, Jun

Ma, Sai

Macdonald, Joanna

Maciejewski, Matthew*

Mahipal, Amit

Mai, Cuc

Mainous, Iii, Arch

Majumdar, Sumit

Malat, Jennifer

Malhotra, Jyoti

Malliarakis, Kate

Mamede, Sílvia

Mancuso, Josephine

Manganello, Jennifer

Manheim, Larry

Manjarrez, Efren

Mann, Devin

Mao, Jun

Marcantonio, Edward

Marcus, Marianne

Markson, Leona

Marusic, Ana

Masilamani, Santhi

Matthias, Marianne

Maunder, Robert

Maurer, Martha

Maxwell, Annette

May, Suepattra

Mayer, Anita

Mcalearney, Ann*

Mccarthy, John

Mccusker, Jane

Mcdonald, Charlotte

Mcinnes, D Keith

Mckinley, Danette*

Mcqueen, Amy

Mcwilliams, J. Michael

Means, Robert

Meerschaert, M. Carmen

Mehrotra, Ateev

Meka, Praveen

Meneghini, Luigi

Merkin, Sharon

Merlin, Jessica*

Metersky, Mark

Methuku, Nanda

Metzger, Maureen

Mhaskar, Rahul

Micco, Guy

Miller, Donald

Min, Lillian

Mintzes, Barbara

Misra, Ranjita 
Mitchell, Matthew*

Mohr, David*

Montello, Martha

Moore, Donald

Mopala, Prashanth

Mor, Vincent*

Morasco, Benjamin

Moreno, Alejandro

Morriss, Richard

Moskowitz, David

Muhlbaier, Lawrence

Mulkey, Zachary

Muniyappa, Kishor

Murarka, Shishir

Musick, David

Mustafa, Reem

Nadkarni, Anagha

Nambisan, Priya

Nass, Sharyl

Nazi, Kim

Nease, Donald

Necheles, Jonathan

Nelson, David

Nelson, David

Nelson, Wendy

Newlin, Kelley

Newman, Lori

Newton, Katherine

Nguyen, Long

Niederdeppe, Jeff

Njike, Valentine

Noel, Polly Hitchcock

Norcini, John

Nunez-Smith, Marcella

Nuru-Jeter, Amani

Nyweide, David

O'leary, Kevin

O'malley, Ann

O'malley, Cheryl

O'malley, Patrick

O'rorke, Jane

Oliver, Brant

Olsen, Maren

Olshansky, Jay

Olson, Douglas

Olson, Sharon*

Opmeer, Brent

Oramasionwu, Christine*

Orlando, Lori

Oudega, Ruud

Overholser, Linda

Oyler, Julie

Ozanne, Elissa

Palmas, Walter

Pandey, Rahul

Panicola, Michael

Papageorgiou, Panos

Papanas, Nikolaos

Pape, Theresa

Paranjape, Anuradha

Parikh, Nina*

Parikh, Puja
Park, Mary Anne

Parnes, Lorne

Patel, Kushang

Patel, Mitesh

Patel, Sunita

Pathak, Ram

Pauker, Stephen

Pendleton, Neil

Penfold, Robert

Penson, David

Pereira, Anne

Perkins, Henry

Perkowski, Linda

Persad, Govind

Persky, Neal

Perwaiz, Muhammad

Pesis-Katz, Irena

Peterson, Cecily

Peterson, George*

Petittii, Diana

Petry, Nancy

Phillips, Erica

Pickard, A. Simon

Picot, Joanna

Pierce, John

Pitts, Stephen

Plantinga, Laura*

Plantz, Diane

Podus, Deborah

Poirier, Paul

Poirier, Paul

Poleshuck, Ellen

Politi, Mary

Pollack, David

Pollack, Harold

Porath, Avi*

Porta, Massimo

Powell, Adam*

Powell, Heidi

Powers, Benjamin

Prentice, Julia

Price, Lori Lyn

Prislin, Michael*

Qureshi, Adnan

Rajan, Sabitha

Rakowski, William

Ramachandra Pai, Rajasree Pai

Ramakrishnan, Kalyanakrishnan

Ramaswamy, Anuradha

Ramaswamy, Megha

Ramesh, Krithi

Rana, Sameer

Randhawa, Gurvaneet

Ranji, Sumant

Ransohoff, David

Rantz, Marilyn

Raue, Patrick

Raza, Shahzad

Razafsha, Mahdi

Reddy, Shalini

Redelmeier, Donald

Reerink, Evert
Reid, Robert

Reis, Shmuel

Reynolds, Grace

Rhoades, Jeffrey

Richards, David

Richter, James

Riesenberg, Lee Ann

Riley, Elise

Rittenhouse, Diane

Rivera Mindt, Monica

Rockwood, Kenneth

Roddy, Juliette

Rodriguez, Carlos

Roebuck, Mark Christopher*

Roetzheim, Richard

Roland, Martin*

Rose, Adam*

Ross, Joseph

Roth, Mary

Rothman, Russell

Rothschild, Jeffrey

Rouf, Emran

Roumie, Christianne

Roy, Christopher

Rubenstein, Lisa

Ruffin, Mack

Ruiz, Jorge

Ruo, Bernice*

Russell, Marcia

Russell, Marcia

Sadler, Anne

Saha, Somnath

Salazar, Sergio

Saliba, Debra

Salmon, Peter

Saluja, Sandeep

Salzman, Gary

Samal, Lipika*

Sanderson, Saskia

Sandhu, Nicole

Santana, Calie

Sanz, Francisco

Sarkar, Urmimala

Sarsour, Khaled

Sayles, Jennifer

Schapira, Marilyn*

Scherrer, Jeffrey

Schim, Stephanie

Schmaltz, Heidi*

Schmidt, John

Schmidt, Shelley

Schmittdiel, Julie

Schneider, John

Schneider, Sandra

Schnelle, John

Schoen, Cathy

Schoenbaum, Stephen

Schoenthaler, Antoinette

Schonberg, Mara

Schumacher, Jessica

Schuwirth, Lambert

Seago, Jean Ann 
Segura, Jennifer

Sehgal, Raj*

Seligman, Hilary

Selim, Magdy

Serumaga, Brian

Sey, Michael

Shah, Minesh

Sharma, Priyanka

Sharma, Rashmi

Shea, Steven*

Shelby, Rebecca*

Shelton, Wayne

Shields, Cleveland

Shireman, Theresa*

Shivakumar, Geetha

Showalter, John*

Shunk, Rebecca

Shuval, Kerem

Silveira, Maria

Siminoff, Laura

Simon, Gregory

Simon, Joel

Simon, Melissa

Sinaiko, Anna

Singer, Eleanor

Skaer, Tracy

Skotzko, Christine

Sliem, Hamdy

Smidth, Margrethe

Smith, Dean

Smith, Robert*

Smith, Steven

Solberg, Leif

Sommers, Elizabeth

Song, John

Sorbero, Melony

Sorkin, Dara

Sorkin, Dara*

Sozio, Stephen

Spang, Healther

Staiger, Tom

Stefan, Mihaela

Steiger, Scott

Steinman, Michael*

Stewart, Susan*

Stoddard, Hugh

Stone, Roslyn

Street, Richard

Strenski, Teri

Stuart, Brad

Stuart, Bruce

Stuckey, Heather

Subhas, Gokulakkrishna

Sudore, Rebecca

Swallow, Nicole

Sweet, David

Tabas, Jeffrey

Tai-Seale, Ming

Tamariz, Leonardo*
Tang, Joyce

Tang, Ning

Tao, Guoyu

Tarn, Derjung

Taylor, William

Telfair, Joseph

Thirumaran, Rajesh

Thorndike, Anne

Thrasher, Angela

Tice, Jeffrey

Tio, Audrey

Tjia, Jennifer

Touchette, Daniel*

Trauth, Jeanette

Triola, Marc

Tsai, Adam

Tsai, Alexander

Tseng, Chien-Wen*

Tuohey, John

Tuot, Delphine*

Tyssen, Reidar

Ubel, Peter

Uchida, Toshiko

Urban, Susan

Vadlamudi, Raja

Vadlamudi, Raja

Vaillancourt, Regis

Van Den Heuvel, Wim

Van Der Molen, Thys

Van Eaton, Erik

Van Schaik, Sandrijn*

Van Scoyoc, Erin*

Varosy, Paul

Vela, Monica

Venkitachalam, Lakshmi*

Venters, Homer

Verma, Ashish

Verma, Sameer

Vijan, Sandeep

Vijayaraghavan, Maya

Viswanathan, Meera

Vogeli, Christine

Voils, Corrine

Vokes, Tamara

Wagner, Dianne

Wagner, James

Wakeman, Sarah

Walker, Edward

Wallace, Robin

Walling, Anne

Wang, Connie

Wang, Jing*

Ward, Kristina

Ward, Lawrence

Ward, Marcia

Warm, Eric

Wassenaar, Michael

Watkins, Katherine

Weiner, Mark
Weiser, Sheri

Weiss, Barry*

Weiss, Carlos*

Weiss, Debora

Weiss, Linda

Welborn, Toney

Weller, Wendy

Werner, Rachel

Wernsing, David

West, Brady

West, Suzanne

White, Mary

White, Richard

Whitley, Elizabeth

Whitney, Simon

Widera, Eric

Wieland, Mark*

Wight, Richard

Wilder, Ron

Wilkin, Noel

Williams, Karen Patricia

Willke, Richard

Wilson, Ira

Wilson, Stephen

Wiseman, Rachel*

Wittich, Christopher

Wolfe, Lisa

Wong, Martin*

Wongsaroj, Patarapha

Wood, Malissa

Woolhandler, Steffie

Woolley, J. Michael

Wright, Lucius

Wulsin, Lawson

Wunderink, Richard

$\mathrm{Xu}, \mathrm{Jin}-\mathrm{Fu}$

$\mathrm{Xu}$, Ruifeng

Yadav, Surinder

Yancy, Jr., William

Yanni, Leanne

Yano, Elizabeth

Yarborough, William

Ye, Lichuan

Yelin, Ed

Yerger, Valerie

Young, Henry

Young, Meredith

Zaas, Aimee

Zema, Carla

Zhang, Xuanping

Zipkin, Daniella*

Zirakzadeh, Ali

Zuberi, Rukhsana

Zubkoff, Lisa*

*Superior Reviewer, defined as $>=2$ reviews, quality score no lower than 4 of 6 on any single review, no review returned past 30 days 